PREPARED FOR SUBMission TO JINST

Light Detection in Noble Elements (LIDINE2017)

22-24 SePTEMBER 2017

SLAC National Accelerator Laboratory

\title{
Simulations for the Global Quantum Efficiency of MicroBooNE Optical Units
}

\author{
S.F. Pate, ${ }^{a, 1}$ T. Wester, ${ }^{b}$ L. Bugel, ${ }^{b}$ J. Conrad, ${ }^{b}$ E. Henderson, ${ }^{a}$ B.J.P. Jones,,${ }^{b, c}$ A.I.L. \\ McLean, ${ }^{a}$ J.S. Moon, ${ }^{b}$ M. Toups, ${ }^{b, d}$ and T. Wongjirad ${ }^{b}$ \\ a Physics Department, New Mexico State University, Las Cruces, NM, 88003, USA \\ ${ }^{b}$ Physics Department, Massachusetts Institute of Technology, Cambridge, MA, 02139, USA \\ ${ }^{c}$ Physics Department, University of Texas at Arlington, Arlington, TX, 76019, USA \\ ${ }^{d}$ Fermi National Accelerator Laboratory, Batavia, IL, 60510, USA \\ E-mail: pate@nmsu.edu
}

KeYwords: Detector alignment and calibration methods (lasers, sources, particle-beams); Time projection chambers; Cryogenic detectors

${ }^{1}$ Corresponding author. 


\section{Contents}

1 Introduction 1

2 Measurements 1

2.1 Absolute Normalization 1

2.2 Radial Dependence 2

3 Simulation 2

4 Results 2

\section{Introduction}

The MicroBooNE detector [1] is a liquid argon time projection chamber currently in operation at Fermi National Accelerator Laboratory. The detector has a light collection system comprised of 32 Hammamatsu R5912-02mod photomultiplier tubes (PMTs), each with an acrylic plate, coated with the wavelength-shifter tetraphenyl butadiene (TPB), placed in front of the photocathode. This combination of a plate-PMT system will be referred to as an "optical unit". These PMTs have an operational quantum efficiency at cryogenic temperatures of $0.153 \pm 0.008$ [1].

We define the global quantum efficiency of an optical unit as the average number of photoelectrons $\left(N_{\mathrm{PE}}\right)$ produced in the photocathode of the PMT for a photon incident on the TPB-coated plate. The global quantum efficiency is a useful calibration factor for MicroBooNE because it enables the use of the light collection system as a trigger and calorimeter.

We have determined the global quantum efficiency for a MicroBooNE optical unit using a simulation model, with two test-stand measurements as a cross-check and validation of the model. The experiments and the model are described in detail in [2].

It is of note that our data and model are consistent with the result for the efficiency of wavelength shifting in TPB for a $1.8 \mu \mathrm{m}$ coating thickness, presented in [3] (figure 17).

\section{Measurements}

\subsection{Absolute Normalization}

The absolute normalization of the global quantum efficiency was obtained through a set of four measurements made in the Bo cryostat at Fermi National Accelerator Laboratory. The measurement procedure is described in detail in [4], and the calibration procedure is described in [2]. The basic setup is repeated here: A polonium-210 alpha source (radius $=2.5 \mathrm{~mm}$ ) is positioned in a stainless steel holder above an optical unit in a liquid argon dewar. The alphas emitted from the source are monoenergetic with energy $5.3 \mathrm{MeV}$ and create $128 \mathrm{~nm}$ scintillation light in the argon, which is converted in the TPB layer of the acrylic plate to visible $(420 \mathrm{~nm})$ light. The converted light is 
collected by the PMT, and the number of photoelectrons is determined. This is repeated for the source holder at two distances (henceforth "near" and "far") from the face of the fixed TPB plate.

\subsection{Radial Dependence}

The efficiency of light collection by an optical unit is known to depend on the radius at which the light hits the acrylic TPB plate. We studied this radial dependence of the global quantum efficiency using a separate test stand and optical unit setup. The specific measurement procedure is outlined in [2], and summarized here: A rod with optical fibers is placed along the radius of the acrylic TPB plate of an optical unit in a liquid argon test stand. The fibers are illuminated with LEDs, and the response of the PMT from each fiber illumination is measured. The functional form of the radial dependence of the PMT response from this test was found to be well-fitted by a Gaussian.

\section{Simulation}

We created a GEANT4 [5] simulation of the test stand used for the absolute normalization study. The simulation is described in detail in [2]. The simulation is built from first principles and incorporates the known optical properties of the test stand materials and the liquid argon. We start by simulating alpha emission from points within the source disk, and ray-tracing the $128 \mathrm{~nm}$ scintillation photons from each alpha position to the TPB plate. We then simulate $420 \mathrm{~nm}$ light emission from the points where $128 \mathrm{~nm}$ photons hit the plate. Finally, we incorporate the efficiencies of the TPB and PMT to determine a simulated number of photoelectrons, $N_{\mathrm{PE}}$, which we compare directly to the absolute normalization measurements.

We check systematic assumptions by re-running the full simulation with randomized parameter combinations, which accounts for our uncertainty in optical and geometric parameters. The results from [2] are reproduced in Figure 1.

We also obtain a reasonable functional form for the radial dependence in agreement with the Gaussian shape observed experimentally. The result from the simulation of the $420 \mathrm{~nm}$ light generated in the acrylic plate, tracked to the PMT photocathode, as an efficiency of photons which reach the PMT per photons generated, is shown in Figure 2. Note that this figure shows only the results of ray tracing photons from the plate to the PMT, i.e. there are no other efficiencies taken into account in this figure.

\section{Results}

We re-state the results from [2] here. The simulation model presented in that work was found to be consistent with the near measurement used to determine the absolute normalization and the radial-dependence of a function which describes the global quantum efficiency at a point on the TPB plate. For the far measurement, we observe that both measurements are in the possible range of simulated values, but fall in the tail of the distribution, and are not consistent with each other. We believe this resulted from purity or temperature instability in the detector introduced while re-filling the cryostat between changing the source holder position. In the future, measurements made in an apparatus with a source holder that could be adjusted without opening the cryostat would be preferred. 

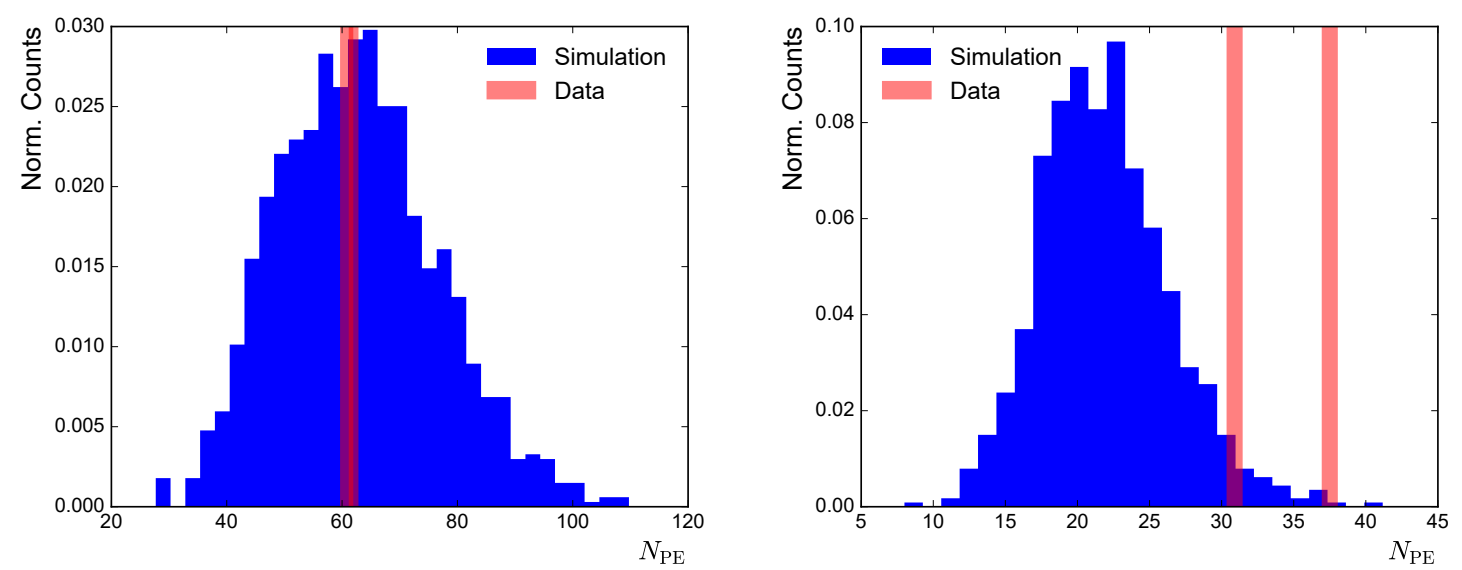

Figure 1. The spread in simulated $N_{\mathrm{PE}}$, compared to the four test stand measurements. The left plot shows the measurements and simulation for the near source configuration, and the right plot shows the measurements and simulation for the far source configuration. Figure reproduced from [2].

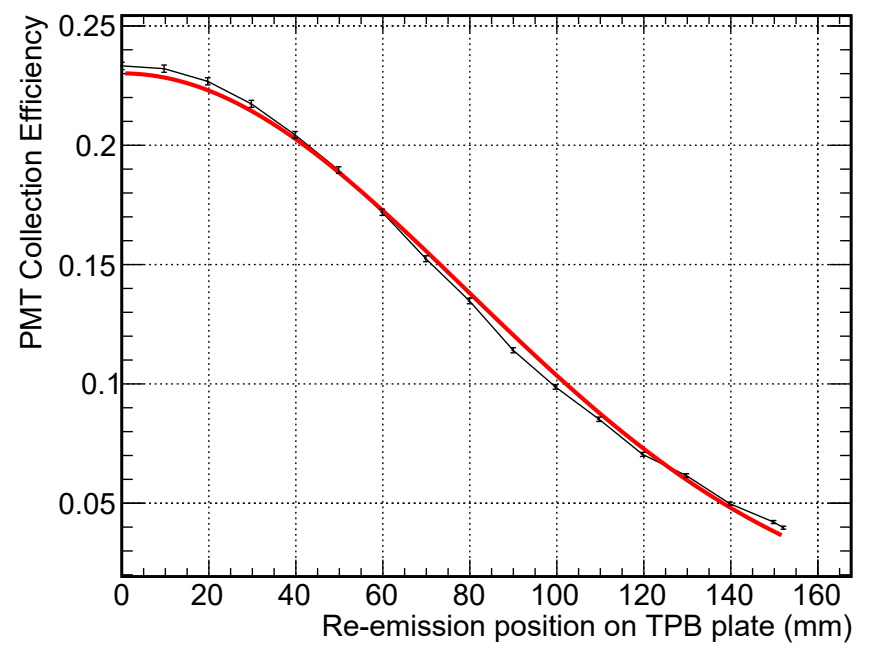

Figure 2. The simulated radial dependence of PMT collection efficiency (black), which is well-described by a Gaussian (red). This figure shows the fraction of $420 \mathrm{~nm}$ photons which originate in the TPB plate that reach the PMT.Figure reproduced from [2]. 
The global quantum efficiency, as averaged over the entire surface of the plate, was found to be $0.0055 \pm 0.0009$. This result was found by integrating over a Gaussian with amplitude equal to the Gaussian from figure 2, and multiplying by the PMT quantum efficiency, re-emission probability in the TPB plate, and correction factors for the temperature and TPB coating thickness. These values and associated uncertainties may be found in [2]. We note that while the model presented is based on the specific MicroBooNE optical unit, the details may be adapted for other detector geometries.

\section{References}

[1] R. Acciarri et al., Design and Construction of the MicroBooNE Detector, JINST 12 (2017) P02017, [1612.05824].

[2] S. Pate et al., A model for the global quantum efficiency for a TPB-based wavelength-shifting system used with photomultiplier tubes in liquid argon in MicroBooNE, 1711.01230.

[3] C. P. Benson, G. D. Orebi Gann and V. M. Gehman, Measurements of the intrinsic quantum efficiency and visible reemission spectrum of tetraphenyl butadiene thin films for incident vacuum ultraviolet light, 1709.05002.

[4] B. J. P. Jones et al., A measurement of the absorption of liquid argon scintillation light by dissolved nitrogen at the part-per-million level, Journal of Instrumentation 8 (2013) P07011.

[5] "Geant4: A toolkit for the simulation of the passage of particles through matter." https://geant4.web.cern.ch. 